Supporting Information

\title{
Iron speciation in organic matter fractions isolated from soils amended with biochar and organic fertilizers
}

Beatrice Giannetta $^{\mathrm{a}}$, César Plaza ${ }^{\mathrm{b}}$, Matthew G. Siebecker ${ }^{\mathrm{c}, \mathrm{d}}$, Giuliana Aquilanti e, Costantino Vischetti a, Jasper R. Plaisier ${ }^{\mathrm{e}}$, Miguel Juanco ${ }^{\mathrm{b}}$, Donald L. Sparks ${ }^{\mathrm{d}}$, Claudio Zaccone f,*

a Department of Agricultural, Food and Environmental Sciences, Polytechnic University of Marche, via Brecce Bianche 10, 60131 Ancona, Italy

b Instituto de Ciencias Agrarias, Consejo Superior de Investigaciones Científicas, Serrano 115 bis, 28006 Madrid, Spain

${ }^{c}$ Department of Plant and Soil Science, Texas Tech University, Lubbock, TX 79409, USA

${ }^{d}$ Department of Plant and Soil Sciences, University of Delaware, Interdisciplinary Science and Engineering (ISE) Laboratory, 221 Academy Street, Newark, Delaware 19716, USA

${ }^{e}$ ELETTRA Sincrotrone Trieste S.C.p.A., S.S. 14 Km 163.5, 34149 Basovizza, Trieste, Italy

${ }^{f}$ Department of Biotechnology, University of Verona, Strada Le Grazie 15, 37134 Verona, Italy

${ }^{*}$ Corresponding author.

E-mail address: claudio.zaccone@univr.it (C. Zaccone)

Pages: 19

Figures: 7

Tables: 4 
Table S1. Total organic $\mathrm{C}$ content, total $\mathrm{N}, \mathrm{C} / \mathrm{N}$ ratio and $\mathrm{Fe}$ concentration (mean \pm pooled standard error) of agricultural soils either unamended (UN), amended with biochar (BC), municipal solid waste compost (MC) and with both of them $(\mathrm{BC}+\mathrm{MC})$.

\begin{tabular}{ccccc}
\hline Soil & $\begin{array}{c}\text { Total organic } \mathbf{C}^{\dagger} \\
\left(g \mathrm{~kg}^{-1}\right)\end{array}$ & $\begin{array}{c}\text { Total N } \\
(\mathrm{g} \mathrm{kg})^{-1}\end{array}$ & $\mathbf{C} / \mathbf{N}^{\dagger}$ & $\begin{array}{c}\mathbf{F e} \\
\left(g \mathrm{~kg}^{-1}\right)\end{array}$ \\
\hline $\mathrm{UN}$ & $11.8 \pm 0.7$ & $1.10 \pm 0.05$ & $10.7 \pm 0.6$ & $22.1 \pm 0.3$ \\
$\mathrm{BC}$ & $19.8 \pm 0.7$ & $1.08 \pm 0.05$ & $18.6 \pm 0.6$ & $22.1 \pm 0.6$ \\
$\mathrm{MC}$ & $14.2 \pm 0.7$ & $1.31 \pm 0.05$ & $10.8 \pm 0.6$ & $21.9 \pm 1.0$ \\
$\mathrm{BC}+\mathrm{MC}$ & $22.6 \pm 0.7$ & $1.42 \pm 0.05$ & $15.9 \pm 0.6$ & $22.7 \pm 0.5$ \\
\hline
\end{tabular}

$\dagger$ Data from Plaza et al. ${ }^{1}$ 

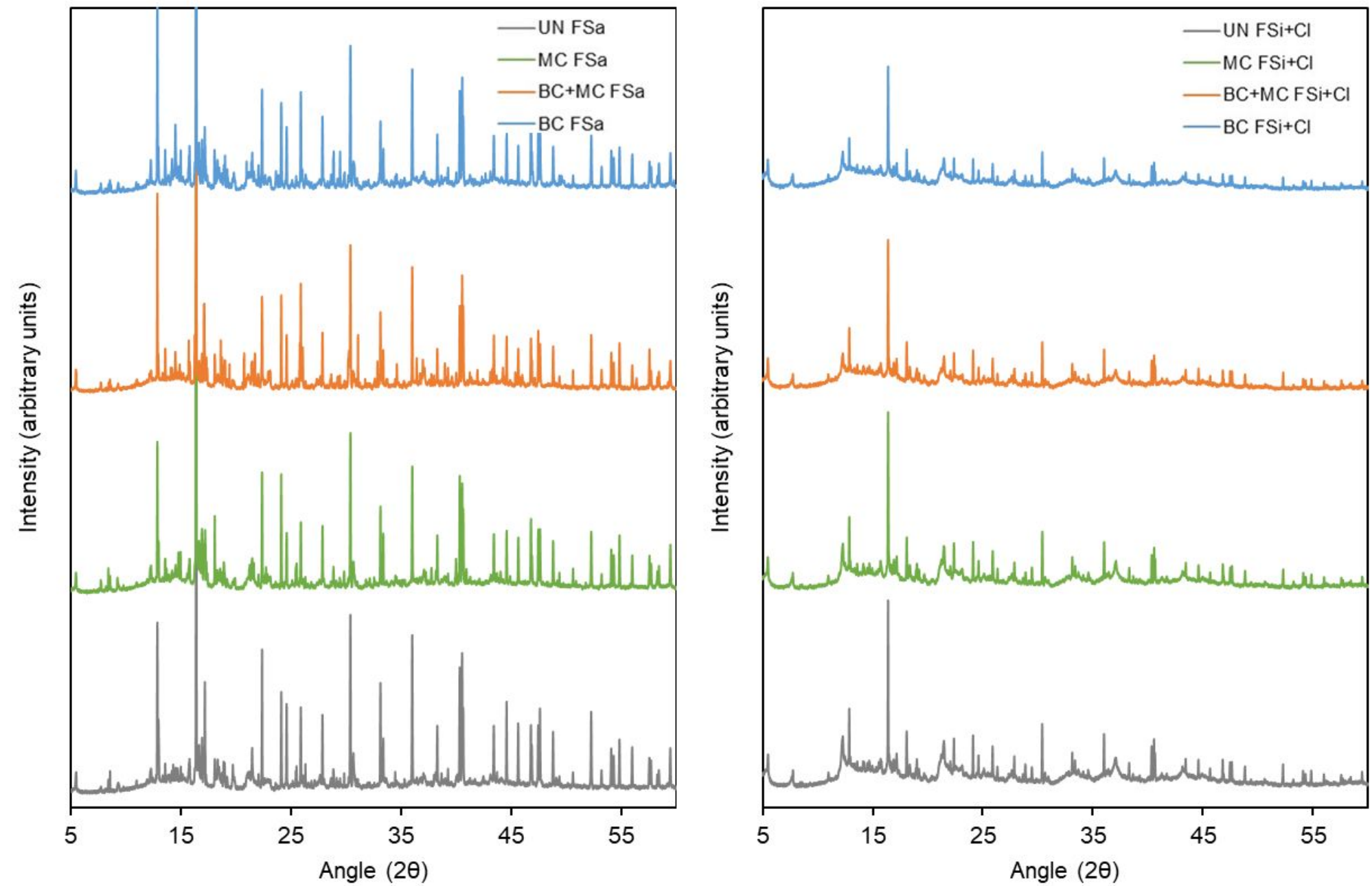

Figure S1. X-ray powder diffractograms of fine sand (FSa) and fine silt and clay $(\mathrm{FSi}+\mathrm{Cl})$ pools of agricultural soils either unfertilized (UN), amended with biochar $(\mathrm{BC})$, municipal solid waste compost $(\mathrm{MC})$ and with both of them $(\mathrm{BC}+\mathrm{MC})$. 


\section{PCA, TT and F-test}

Principal component analysis is used in LCF of EXAFS data to determine the number of standards to use in the fitting. It is important to use an appropriate number of standards so that minor components or false positive results are avoided. The results of PCA are summarized in Table S2 and Figure S2. Sixpack software was used to carry out PCA of the EXAFS data. ${ }^{2}$

In a first step, the Factor Indicator Function, ${ }^{3}$ the IND value obtained from the PCA analysis, reached the minimum for four components, indicating that four was the ideal number of structurally meaningful components. Moreover, the scree plot represented in Figure S2 indicates a break in the slope at two components, indicating that two components would be necessary to adequately reconstruct the sample EXAFS data using the standards.

In the second step, the specific components to be included in the LCF were selected by TT, using the SPOIL function. ${ }^{4}$ The SPOIL values were $<1.5$ (Table S3) and most of the models classified as excellent model compounds, the best fit of unamended and amended samples was obtained using three fitting components. The description of the standards used for LCF is reported in Table S3. All the standards have previously published and are described in O'Day et al. ${ }^{5}$ Chi-square, R-values, and SPOIL and chi-square are also reported. The appendix 1 of O'Day et al..$^{5}$ contains the complete description of the sources of the standards used.

F-tests (or Hamilton tests) were carried out on LCF results with different numbers of standards to determine if the addition of a new standard significantly improved the fit. Ftest values were calculated using a regularized lower incomplete beta function calculator. For the F-test, a 95\% confidence level was implemented. By using this cutoff value, there 
is $<5 \%$ probability that improvement in the fit is caused by random variation or noise in the data.

The F-test value of $n+1$, where $n$ is the number of EXAFS standards used in LCF, is the probability that improvement in the fit with one additional standard is related to fitting noise in the data. For example, for a fit with two standards versus a fit with three standards, an F-test value of $30 \%$ means there is a $30 \%$ probability that fit improvement is due to noise. Adding more standards to a linear combination fit generally reduces the R-factor value of the fit, so the F-test provides quantitative data indicating if fit improvement is statistically significant or not. 
Table S2. Results of the Principal Component Analysis (PCA) performed on the EXAFS spectra from the 8 samples analyzed.

\begin{tabular}{ccccc}
\hline Component & Eingenvalue & Variance & Cum. Var. $^{\mathbf{a}}$ & IND $^{\mathbf{b}}$ \\
\hline 1 & 55.771 & 0.949 & 0.949 & 0.01468 \\
2 & 1.766 & 0.030 & 0.979 & 0.00803 \\
3 & 0.615 & 0.010 & 0.990 & 0.00629 \\
4 & 0.325 & 0.005 & 0.995 & 0.00420 \\
5 & 0.097 & 0.001 & 0.997 & 0.00594 \\
6 & 0.078 & 0.001 & 0.998 & 0.00874 \\
7 & 0.039 & 0.000 & 0.990 & 0.02939 \\
8 & 0.029 & 0.000 & 1.0 & NA \\
\hline
\end{tabular}

${ }^{a}$ Cumulative variance.

${ }^{b}$ Indicator function. ${ }^{3}$ 
Table S3. EXAFS standard used for LCF. Target transformation was applied to determine SPOIL values, chi square and R-values of each standard considered. Source: O'Day et al. ${ }^{5}$

\begin{tabular}{crcc}
\hline Standard & Chi sq & R & SPOIL $^{\mathbf{a}}$ \\
\hline Fe (III) citrate & 162.37 & 0.10 & 0.44 \\
Fe (III) ACAC & 348.77 & 0.14 & 0.48 \\
Ferrihydrite & 165.81 & 0.15 & 0.46 \\
Goethite & 296.95 & 0.19 & 1.09 \\
Hematite & 1397.66 & 0.53 & 0.83 \\
Magnetite & 962.75 & 0.59 & 2.30 \\
Siderite & 701.31 & 0.74 & 0.76 \\
Illite & 76.39 & 0.62 & 0.82 \\
Nontronite & 203.87 & 0.09 & 1.40 \\
Smectite & 200.05 & 0.08 & 0.38 \\
Vermiculite & 401.70 & 0.27 & 0.56 \\
Chlorite & 354.93 & 0.26 & 0.06 \\
\hline
\end{tabular}

${ }^{a}$ SPOIL classification: 0-1.5, excellent; 1.5-3 good; 3-4.5, fair; 4.5-6, acceptable, and >6, unacceptable reference. ${ }^{4}$ 
Table S4. Summary of Fe mineralogy of fine sand (FSa) and fine silt and clay $(\mathrm{FSi}+\mathrm{Cl})$ pools of agricultural soils either unamended (UN), amended with biochar (BC), municipal solid waste compost $(\mathrm{MC})$ and with both of them $(\mathrm{BC}+\mathrm{MC})$ based on EXAFS linear combination fitting over the $k$ range 2.5-10 $\AA$. There were 15.3 independent points (measurements) in the LCF.

\begin{tabular}{ccccr}
\hline Sample & Chlorite & Ferrihydrite & Fe(III)-citrate & \multicolumn{1}{c}{ Sum } \\
\hline UN FSi+Cl & $22 \%$ & $48 \%$ & $37 \%$ & $107 \%$ \\
UN FSa & $26 \%$ & $44 \%$ & $18 \%$ & $89 \%$ \\
BC FSi+Cl & $21 \%$ & $47 \%$ & $44 \%$ & $112 \%$ \\
BC FSa & $36 \%$ & $29 \%$ & $31 \%$ & $96 \%$ \\
$\mathrm{MC} \mathrm{FSi}+\mathrm{Cl}$ & $18 \%$ & $45 \%$ & $43 \%$ & $105 \%$ \\
$\mathrm{MC} \mathrm{FSa}$ & $31 \%$ & $28 \%$ & $31 \%$ & $90 \%$ \\
$\mathrm{BC}+\mathrm{MC} \mathrm{FSi}+\mathrm{Cl}$ & $19 \%$ & $46 \%$ & $41 \%$ & $107 \%$ \\
$\mathrm{BC}+\mathrm{MC} \mathrm{FSa}$ & $25 \%$ & $28 \%$ & $35 \%$ & $88 \%$ \\
\hline
\end{tabular}


Figure S2. Principal component analysis (PCA) results. The scree plot shows a break in slope at two components.

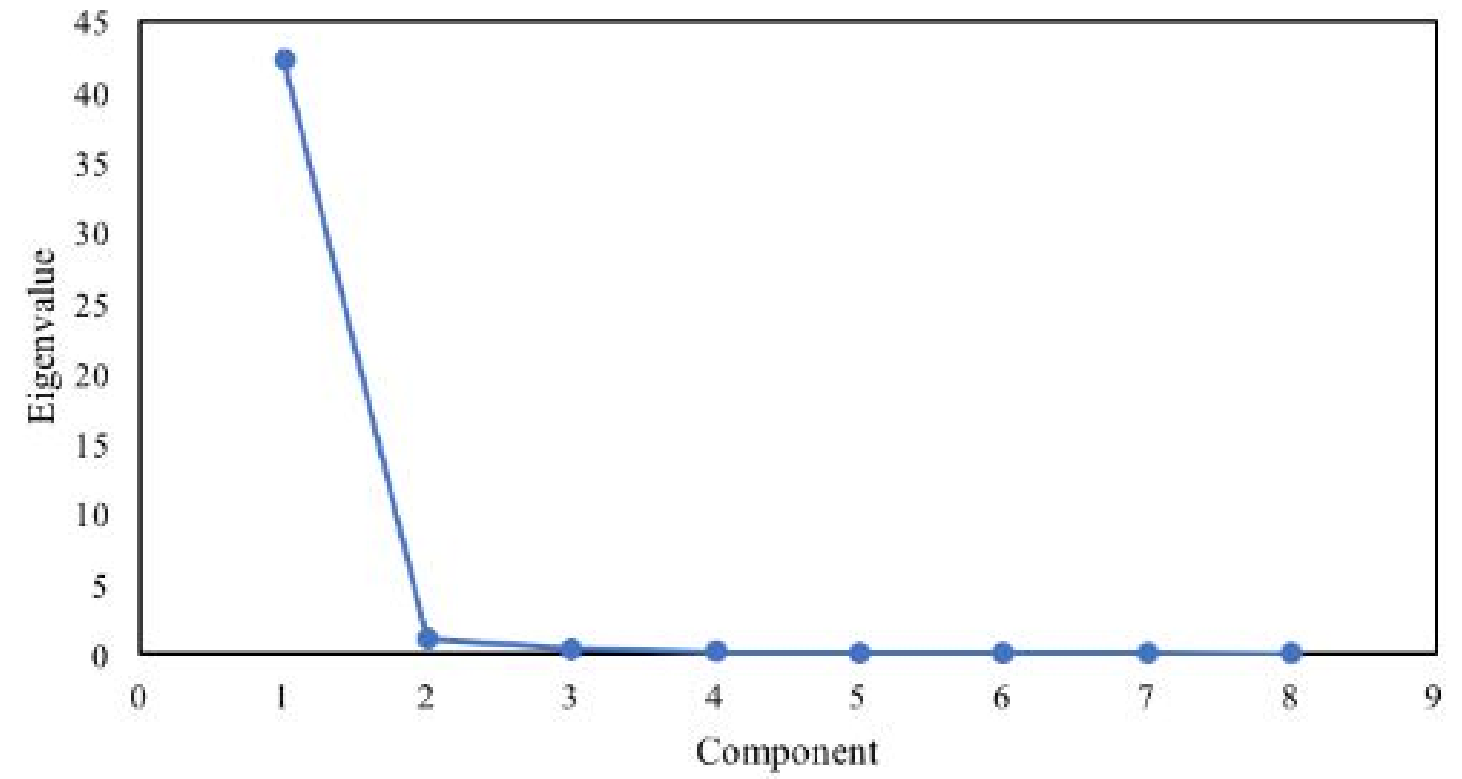


Figure S3. EXAFS spectra of the standards considered for LCF of the soil fractions. Sources and descriptions of each standard are indicated in Table S3.

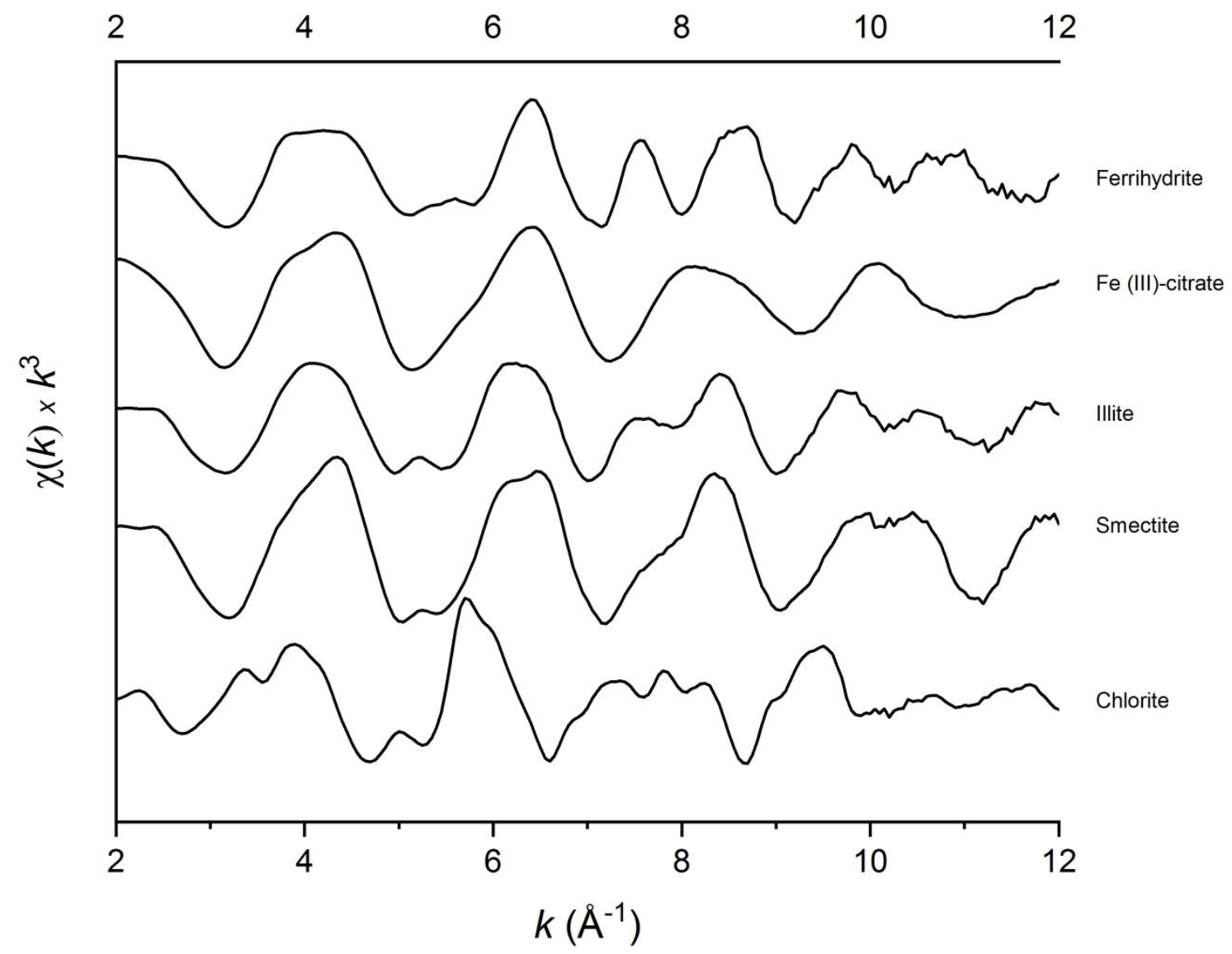


Figure S4. Fourier transform (FT) spectra of the standards considered for LCF of the soil fractions. Sources and descriptions of each standard are indicated in Table S3.

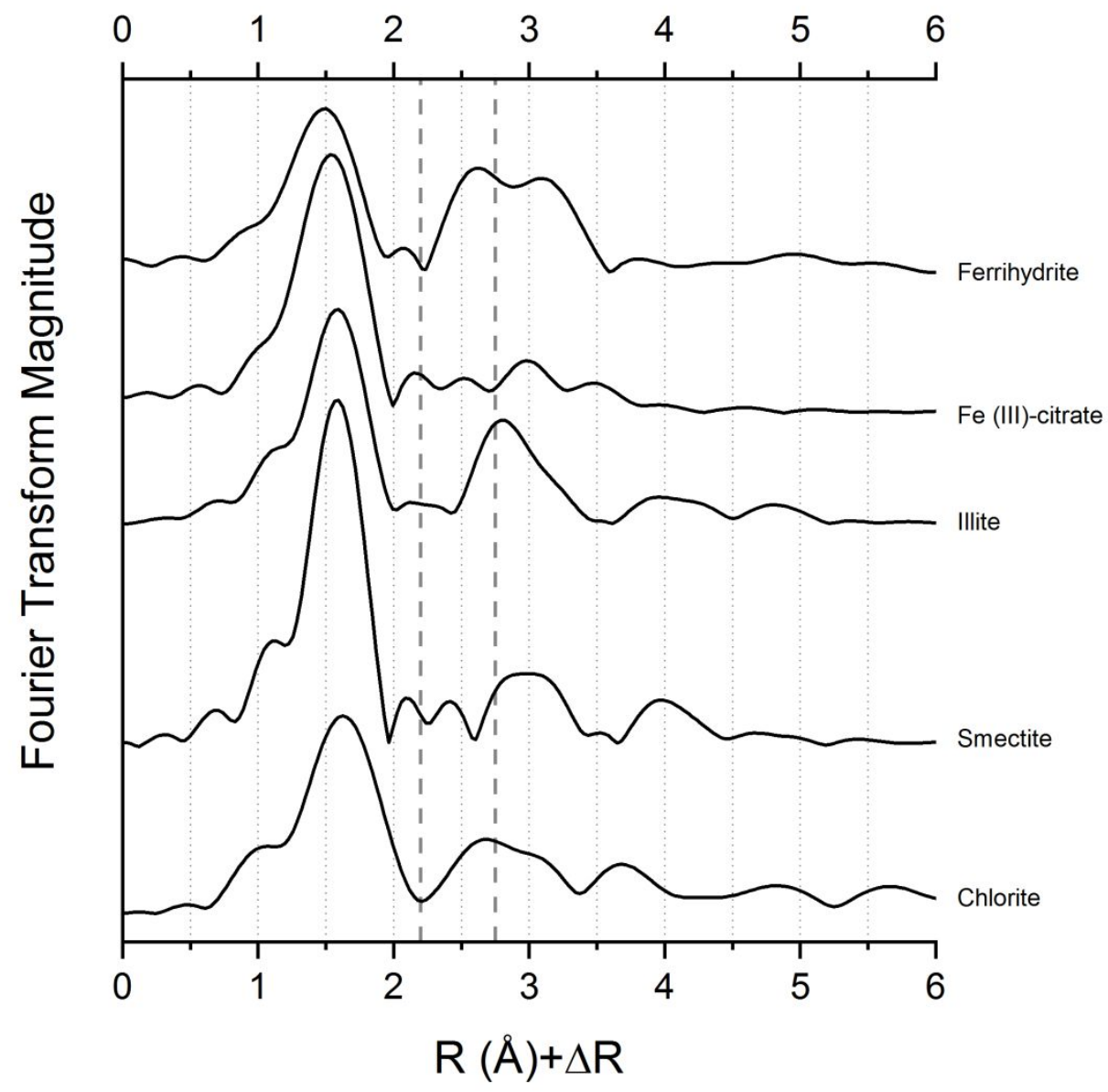


Figure S5. Fourier transform (FT) spectra of the fine silt and clay $(\mathrm{FSi}+\mathrm{Cl})$ and fine sand (FSa) fractions of agricultural soils either unamended (UN), amended with biochar (BC), municipal solid waste compost (MC) and with both of them (BC+MC).

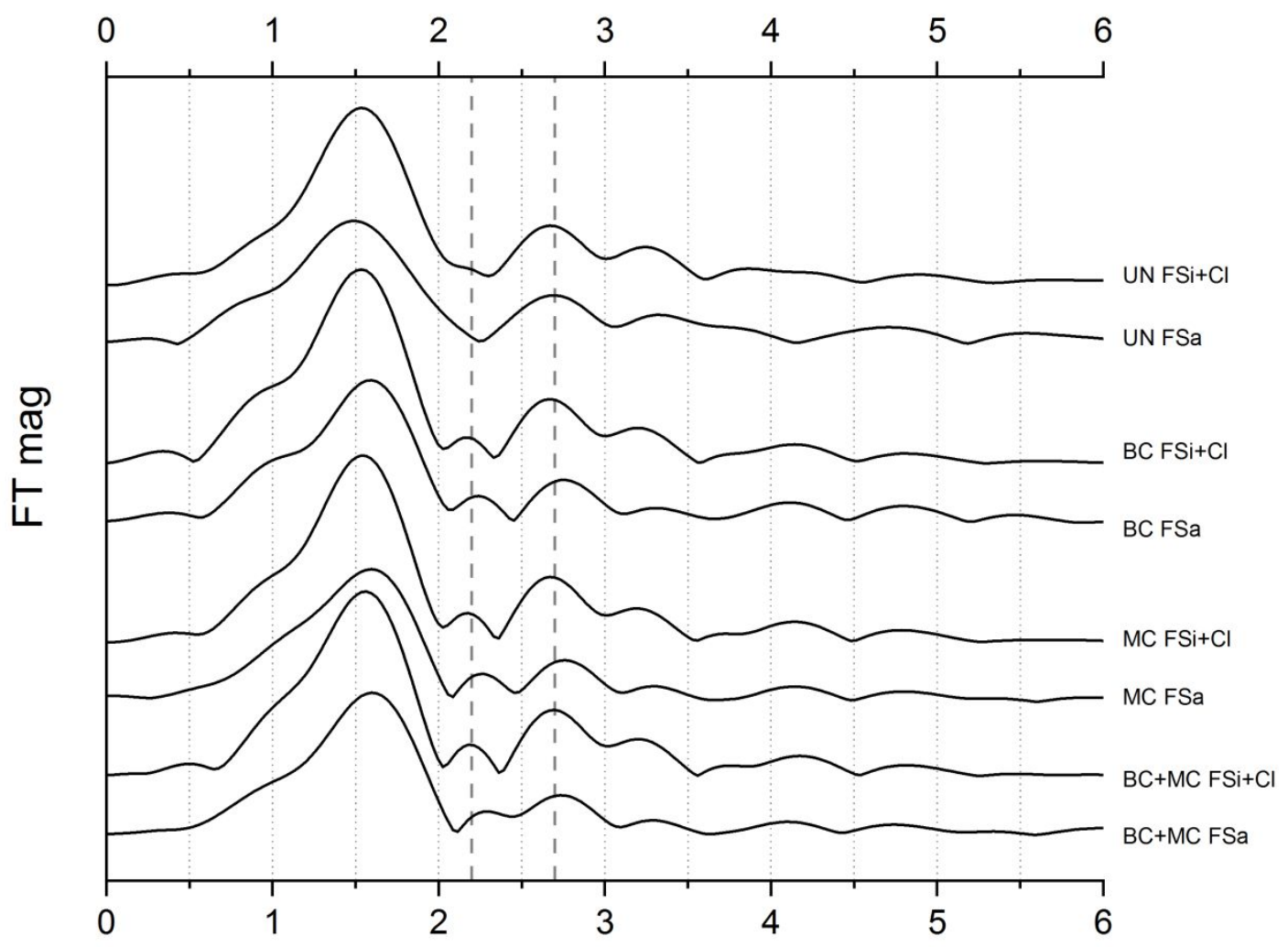




\section{Influence of the selection of $\eta$ and $\sigma$ parameters}

In order to distinguish how modifications in the wavelet parameters affect the resulting plot, $\eta$ was changed from 9 to 4 (Figures S6 and S7). This modification is useful to analyze higher resolution in k-space versus in R-space or vice versa to understand where different scattering maxima migrate while the $\eta / \sigma$ ratio changes. ${ }^{6}$ At $\eta=9$, the resolution in R-space is high while resolution in k-space is low, reaching the best spatial resolution in R-space at $\eta=7$ (Figures S6 and S7). The scattering maxima between 6 and $10 \AA^{-1}$ starts to be more clearly resolved at $\eta=7$, with the best resolution at $\eta=6$. At $\eta=7$, the maximum starts to separate into discrete peaks for both standards (Figure S6) and samples (Figure S7); this split indicates the major component is likely composed of two minor components. In all amended $\mathrm{FSi}+\mathrm{Cl}$ fractions, the peak resolution over the $\eta$ range is similar to the one in ferrihydrite (Figures S6 and S7 a-d). Conversely, in FSa, the plots present clear similarity with the WT plots of Fe(III)-citrate (Figure S6). It is noticeable the absence of the Fe shell with a strong feature at 7-8 $\AA^{-1}$ and $2.75 \AA$, typical of the Fe(III) citrate standard. Additionally, changing $\eta$ from 9 to 1 , the evolution of scattering maxima at 7 and $10 \AA^{-1}$ typical of the Fe(III) citrate standard (Figure S6) is also evident in all amended FSa fractions (Figure S7 b-d), but not in the unamended one (Figure S7a). 
Figure S6. WT plots illustrating the effect of changes to the eta ( $\eta$ ) parameter on the second and third shells of the standards. In this series of plots, $\sigma$ remains constant while $\eta$ ranges from 9 to 4 .

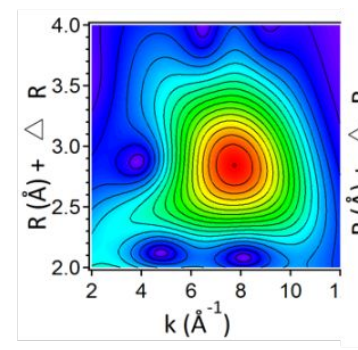

Ferrihydrite $\eta=9, \sigma=1$

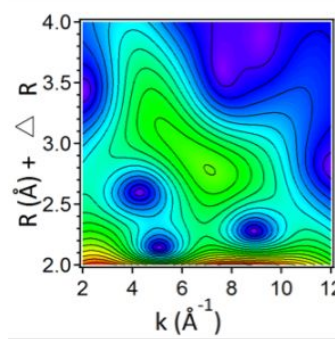

Fe (III)-citrate $\eta=9, \sigma=1$

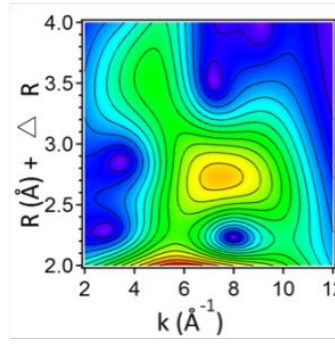

Chlorite $\eta=9, \sigma=1$

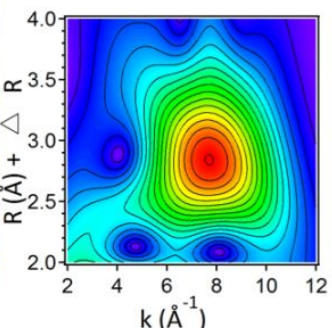

Ferrihydrite $\eta=8, \sigma=1$

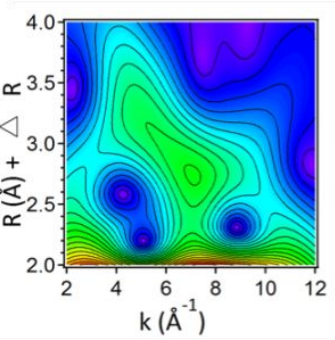

Fe (III)-citrate $\eta=8, \sigma=1$

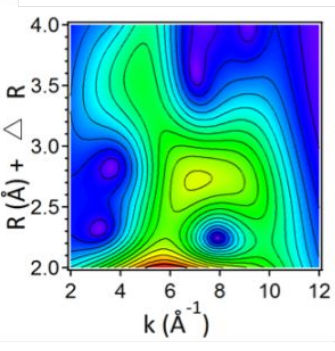

Chlorite $\eta=8, \sigma=1$

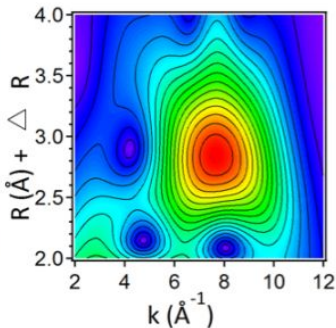

Ferrihydrite $\eta=7, \sigma=1$

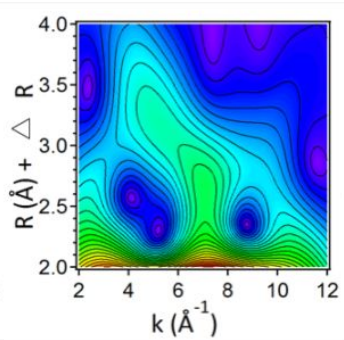

Fe (III)-citrate $\eta=7, \sigma=1$

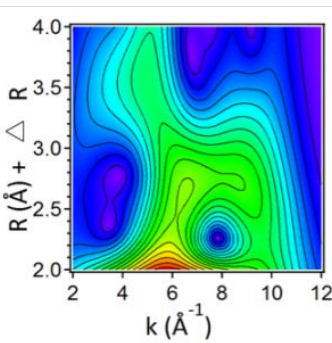

Chlorite $\eta=7, \sigma=1$

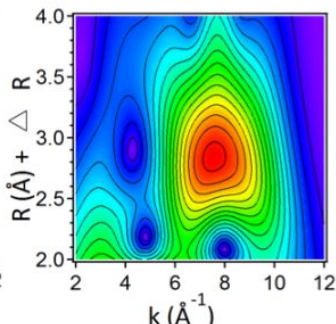

Ferrihydrite $\eta=6, \sigma=1$

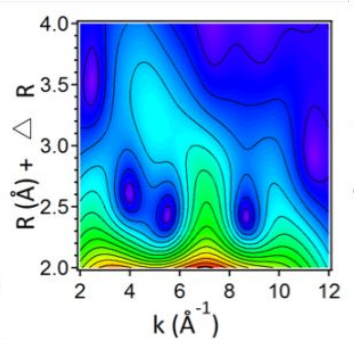

Fe (III)-citrate $\eta=6, \sigma=1$

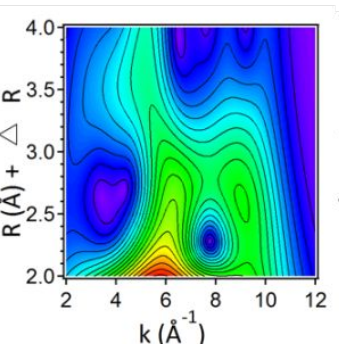

Chlorite $\eta=6, \sigma=1$

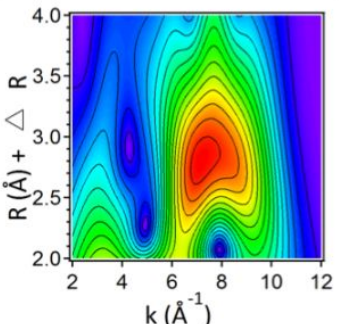

Ferrihydrite $\eta=5, \sigma=1$

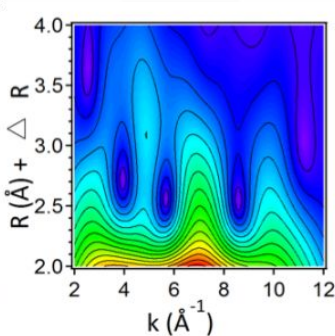

Fe (III)-citrate $\eta=\mathbf{5}, \boldsymbol{\sigma}=\mathbf{1}$

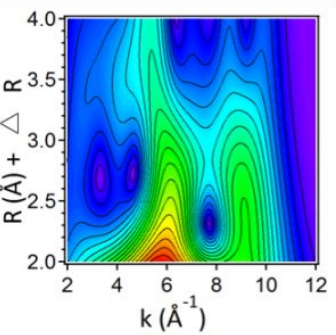

Chlorite $\eta=5, \sigma=1$

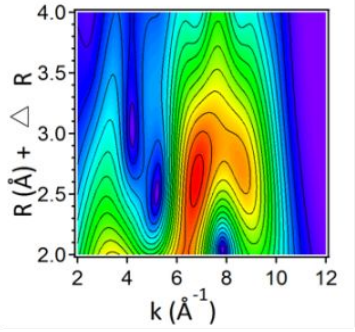

Ferrihydrite $\eta=4, \sigma=1$

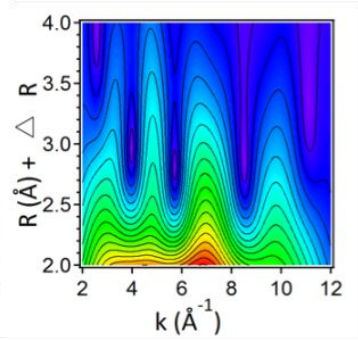

Fe (III)-citrate $\eta=4, \sigma=1$

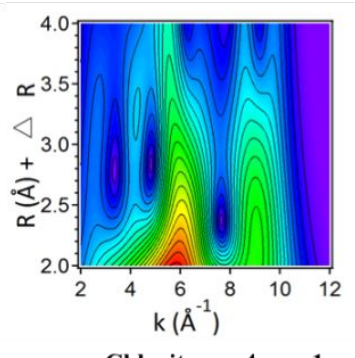




\section{Figure S7}

WT plots illustrating the effect of changes to the eta ( $\mathrm{\eta}$ ) parameter on the second and third shells of the fine silt and clay (FSi+Cl) and fine sand (FSa) fractions of (a) agricultural soils either unamended (UN), (b) amended with biochar (BC), (c) municipal solid waste compost (MC) and (d) with both of them $(\mathrm{BC}+\mathrm{MC})$. In this series of plots, $\sigma$ remains constant while $\eta$ ranges from 9 to 4 .
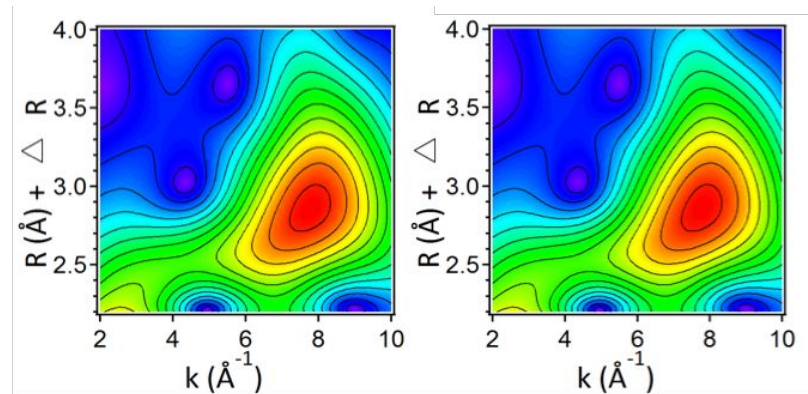

$\mathrm{UN} \mathrm{FSi}+\mathrm{Cl} \eta=9, \sigma=1$

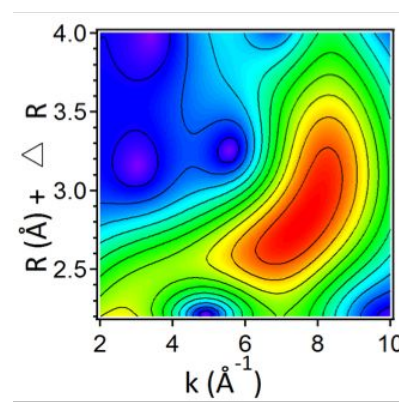

UN FSa $\eta=9, \sigma=1$
$\mathrm{UN} \mathrm{FSi}+\mathrm{Cl} \eta=8, \sigma=1$

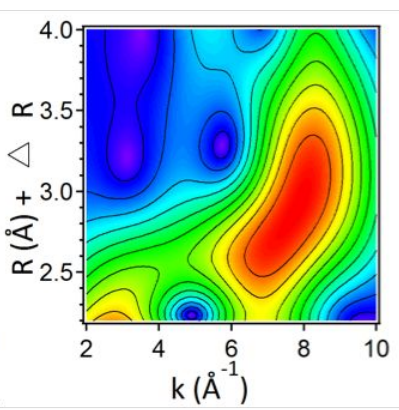

UN FSa $\eta=8, \sigma=1$

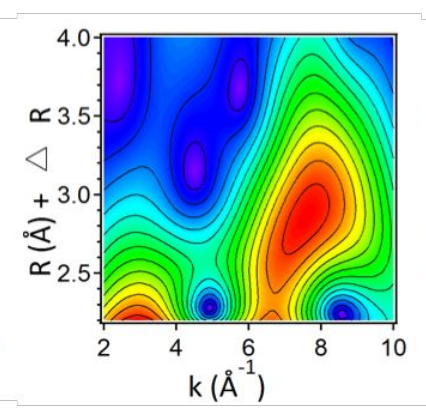

$\mathrm{UN} \mathrm{FSi+Cl} \eta=7, \sigma=1$

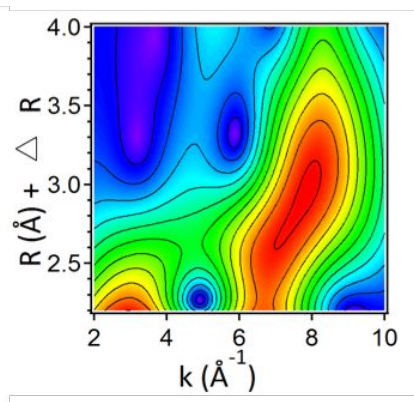

UN FSa $\eta=7, \sigma=1$

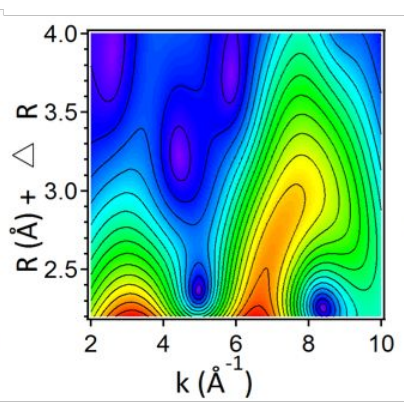

$\mathrm{UN} \mathrm{FSi+Cl} \eta=6, \sigma=1$

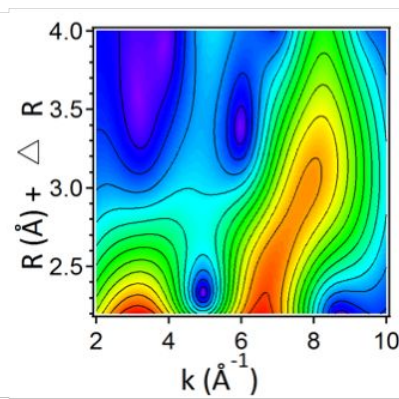

$\mathrm{UN} \mathrm{FSa} \eta=6, \sigma=1$

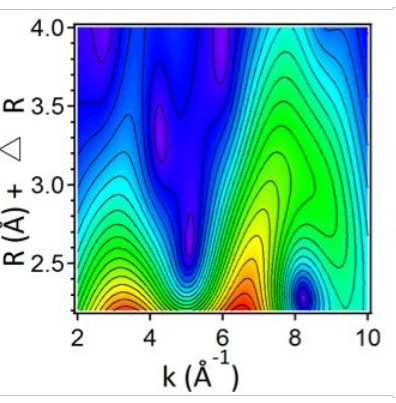

$\mathrm{UN} \mathrm{FSi}+\mathrm{Cl} \eta=5, \sigma=1$

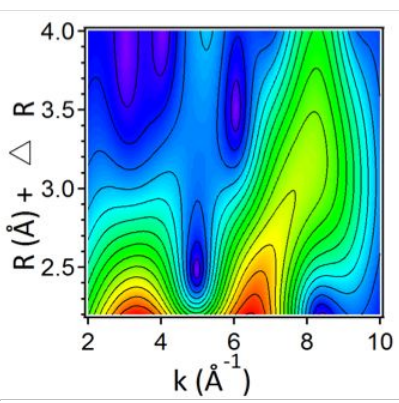

UN FSa $\eta=5, \sigma=1$

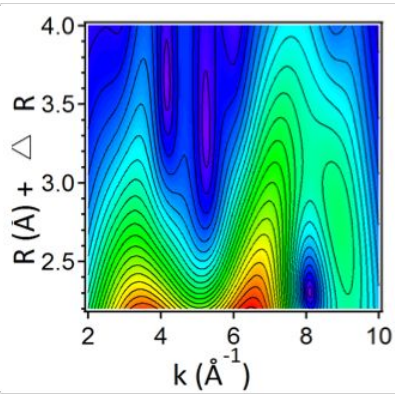

$\mathrm{UN} \mathrm{FSi}+\mathrm{Cl} \eta=4, \sigma=1$

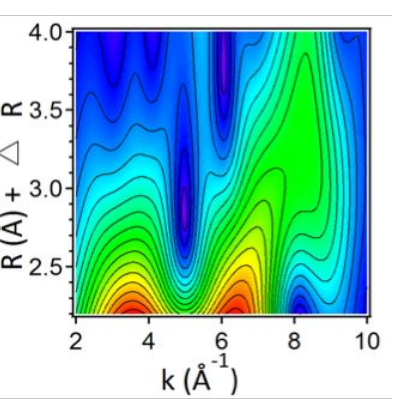

UN FSa $\eta=4, \sigma=1$

a) 


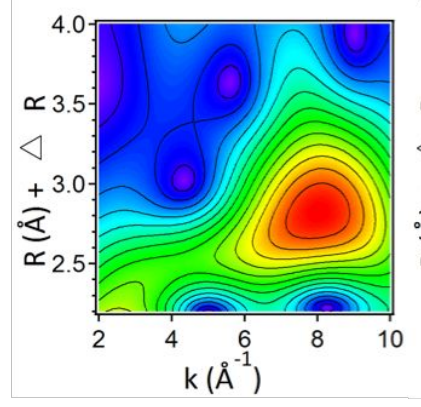

$\mathrm{BC} \mathrm{FSi}+\mathrm{Cl} \eta=9, \sigma=1$

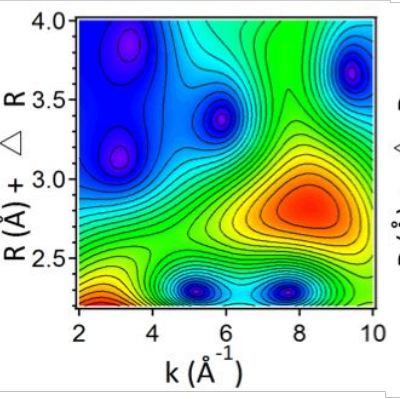

BC FSa $\eta=9, \sigma=1$

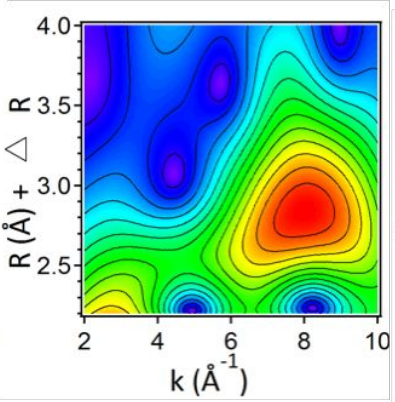

$\mathrm{BC} \mathrm{FSi}+\mathrm{Cl} \eta=8, \sigma=1$

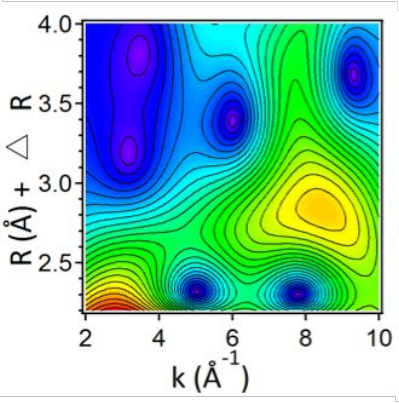

BC FSa $\eta=8, \sigma=1$

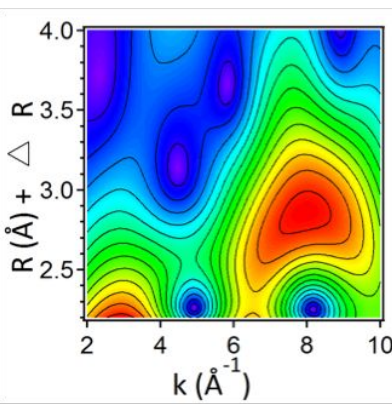

$\mathrm{BC} \mathrm{FSi+Cl} \eta=7, \sigma=1$

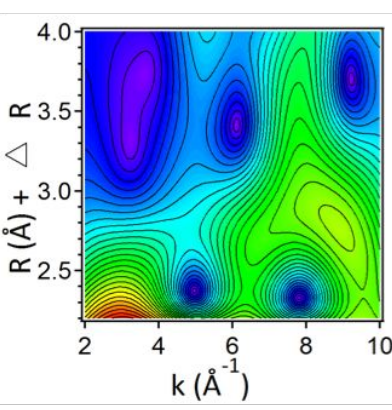

BC FSa $\eta=7, \sigma=1$

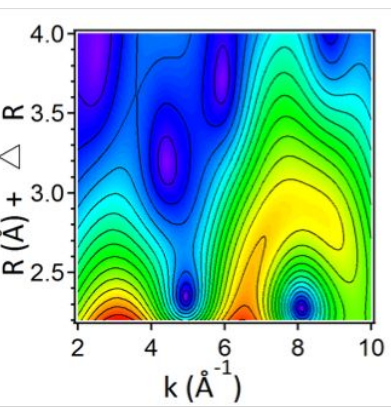

$\mathrm{BC} \mathrm{FSi+Cl \eta}=6, \sigma=1$

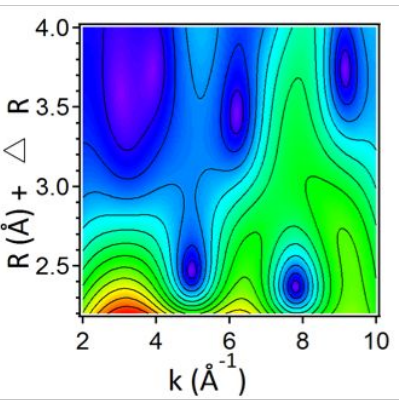

BC FSa $\eta=6, \sigma=1$

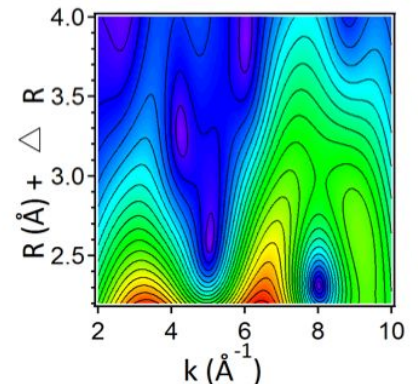

$\mathrm{BC} \mathrm{FSi+Cl} \eta=5, \sigma=1$

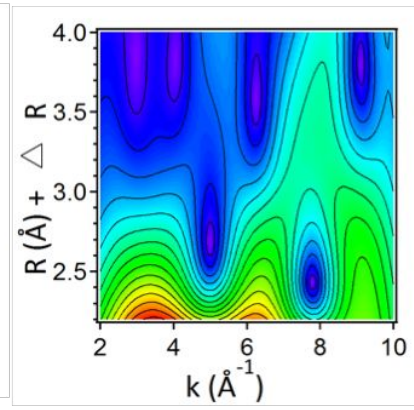

BC FSa $\eta=5, \sigma=1$

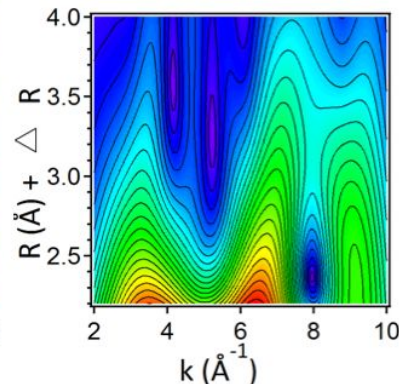

$\mathrm{BC} \mathrm{FSi}+\mathrm{Cl} \eta=4, \sigma=1$

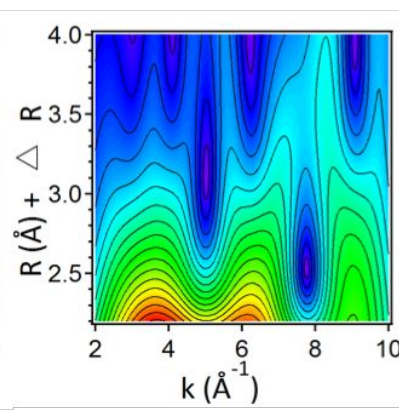

BC FSa $\eta=4, \sigma=1$

b) 

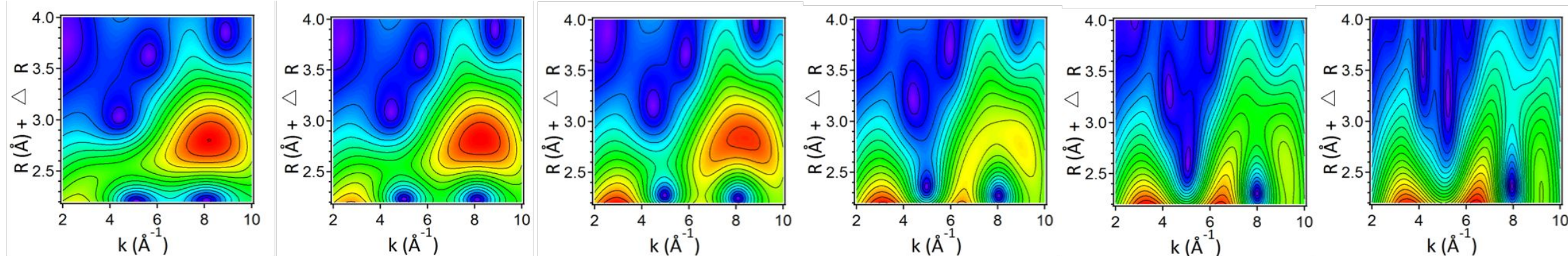

$\mathrm{MC} \mathrm{FSi}+\mathrm{Cl} \eta=9, \sigma=1$

$\mathrm{MC} \mathrm{FSi}+\mathrm{Cl} \eta=8, \sigma=1$

$\mathrm{MC} \mathrm{FSi}+\mathrm{Cl} \eta=7, \sigma=1$

$\mathrm{MC} \mathrm{FSi}+\mathrm{Cl} \eta=6, \sigma=1$

$\mathrm{MC} \mathrm{FSi}+\mathrm{Cl} \eta=5, \sigma=1$

$\mathrm{MC} \mathrm{FSi}+\mathrm{Cl} \eta=4, \sigma=1$
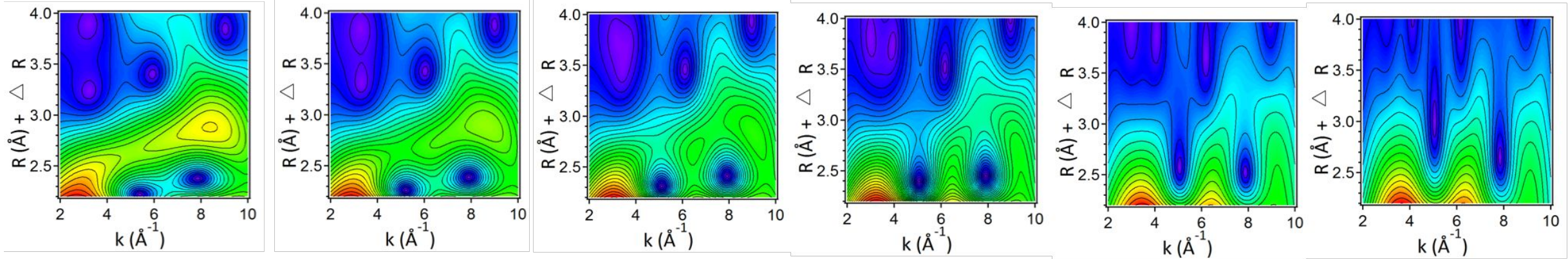

MC FSa $\eta=9, \sigma=1$

MC FSa $\eta=8, \sigma=1$

MC FSa $\eta=7, \sigma=1$

MC FSa $\eta=6, \sigma=1$

$\operatorname{MC~FSa~} \eta=5, \sigma=1$

MC FSa $\eta=4, \sigma=1$

c) 


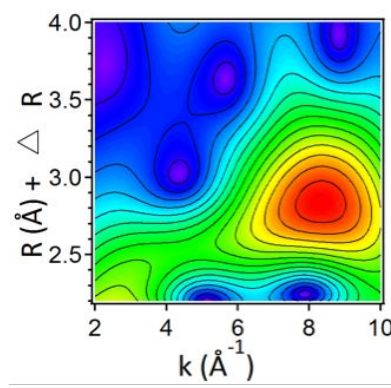

$\mathrm{BC}+\mathrm{MC} \mathrm{FSi}+\mathrm{Cl} \eta=9, \sigma=1$

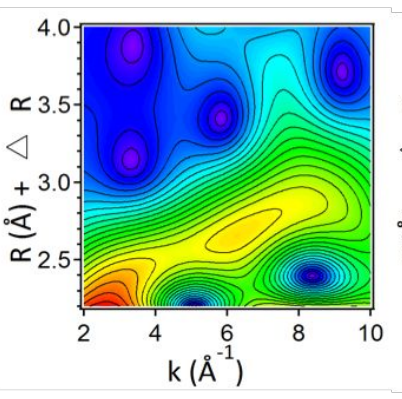

BC+MC FSa $\eta=9, \sigma=1$

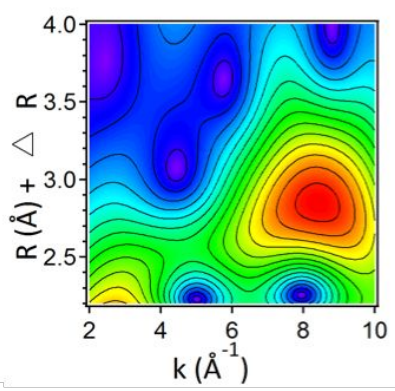

$\mathrm{BC}+\mathrm{MC} \mathrm{FSi}+\mathrm{Cl} \eta=8, \sigma=$

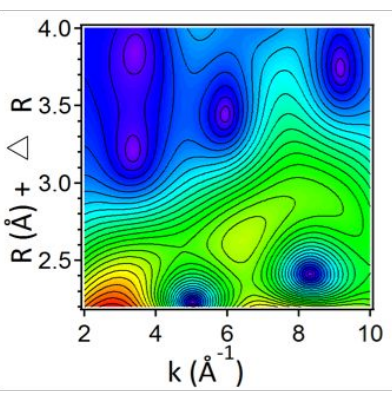

$\mathrm{BC}+\mathrm{MC}$ FSa $\eta=8, \sigma=1$

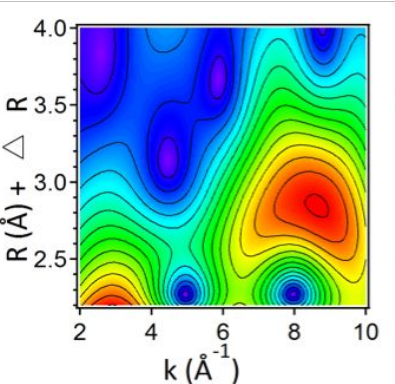

$\mathrm{BC}+\mathrm{MC} \mathrm{FSi}+\mathrm{Cl} \eta=7, \sigma=1$

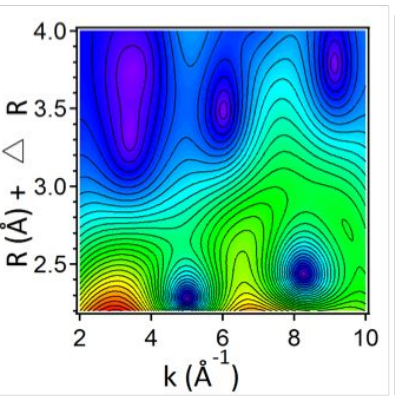

BC+MC FSa $\eta=7, \sigma=1$

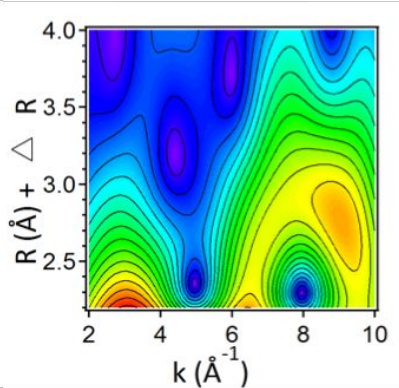

$\mathrm{BC}+\mathrm{MC} \mathrm{FSi}+\mathrm{Cl} \eta=6, \sigma=$

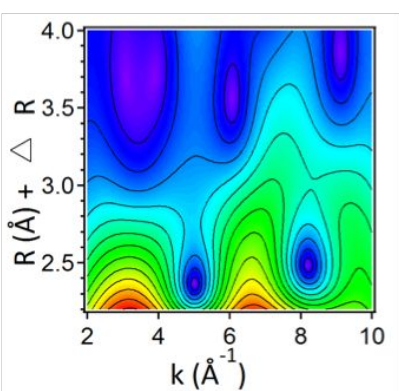

$B C+M C F S a \eta=6, \sigma=1$

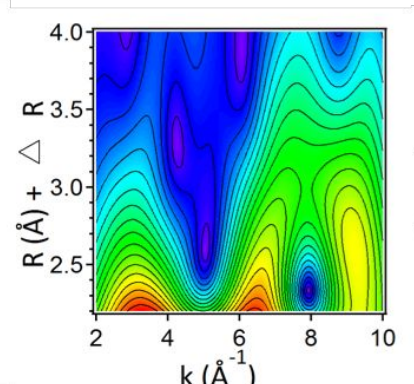

$\mathrm{BC}+\mathrm{MC} \mathrm{FSi}+\mathrm{Cl} \eta=5, \sigma=1$

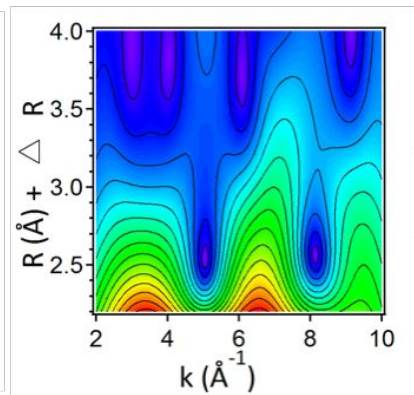

$\mathrm{BC}+\mathrm{MC} \mathrm{FSa} \eta=5, \sigma=1$

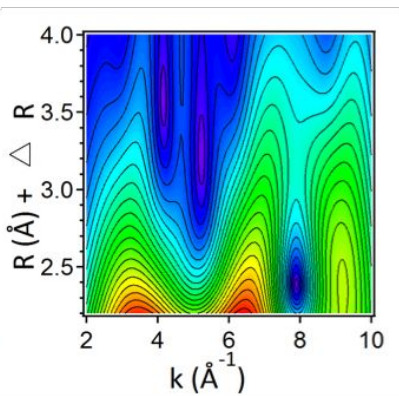

$\mathrm{BC}+\mathrm{MC} \mathrm{FSi}+\mathrm{Cl} \eta=4, \sigma=$

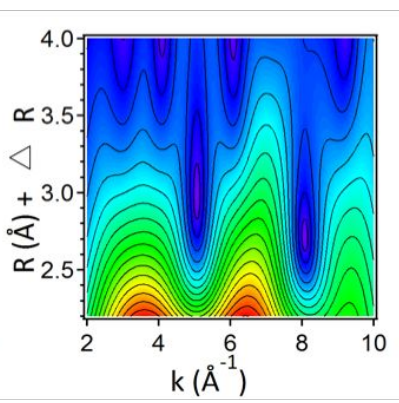

$\mathrm{BC}+\mathrm{MC}$ FSa $\eta=4, \sigma=1$

d) 


\section{References}

(1) Plaza, C.; Giannetta, B.; Fernández, J. M.; López-de-Sá, E. G.; Polo, A.; Gascó, G.; Méndez, A.; Zaccone, C. Response of different soil organic matter pools to biochar and organic fertilizers. Agric. Ecosyst. Environ. 2016, 225, 150-159.

(2) Webb, S. M. SIXPack: A graphical user interface for XAS analysis using IFEFFIT. Phys. Scr. 2005, T115, 1011-1014.

(3) Malinowski, E. R. Determination of the number of factors and the experimental error in a data matrix. Anal. Chem. 1977, 49, 612-617.

(4) Malinowski, E. R. Theory of error for target factor analysis with applications to mass spectrometry and nuclear magnetic resonance spectrometry. Anal. Chim. Acta 1978, 103, 339354.

(5) O’Day, P. A.; Rivera, N.; Root, R.; Carroll, S. A. X-ray absorption spectroscopic study of Fe reference compounds for the analysis of natural sediments. Am. Mineral. 2004, 89, 572-585.

(6) Siebecker, M. G.; Sparks, D. L. Structural differentiation between layered single (Ni) and double metal hydroxides (Ni-Al LDHs) using wavelet transformation. J. Phys. Chem. A 2017, $121,6992-6999$. 